\title{
Tracking the Evolution of in-situ Radiochemistry with Transmission Electron Microscopy
}

\author{
J. A. Aguiar*, L. Wolfsberg, W. Taylor, B. P. Uberuaga, B. Scott, C.R. Stanek
}

Los Alamos National Laboratory, Los Alamos, NM, 80465

${ }^{*}$ Now at, National Renewable Energy Laboratory, Golden, CO 80401

Recent developments within the nuclear materials community have lead researchers to predict nanocrystalline materials can plausibly address concerns regarding nucleation, growth, migration, waste storage, and fission product evolution at higher temperatures and radiation environments. From the work so far in the community, it is not clear how these phases of material impact waste form evolution and behavior. Advanced microscopy allows for unprecedented insights into the underlying physical mechanisms responsible for the improved performance of these materials, especially through the use and study of evolving decaying isotopes. To extend our understanding of nuclear materials after service, in particular waste forms, we have studied the fundamental evolution of these materials induced by the decay of radioisotopes by coupling the use of accelerated aging and analytical microscopy.

The effect of transmutation of radionuclides, especially "short-lived" Sr-90 and Cs-137, to chemically distinct daughter products ( $\mathrm{Zr}$ and Ba respectively) will impact nuclear waste form stability. Due to the technical challenges associated with studying this problem, the topic of transmutation has received limited attention during the past 30 years of waste form development. In order to develop a predictive capability to design radiation tolerant and chemically robust nuclear waste forms, we must first address a fundamental materials science question, namely what is the impact of daughter product formation on the stability of solids comprised of radioactive isotopes. To address this question, a multidisciplinary approach integrating first principles modeling with the synthesis and characterization of small, highly radioactive surrogate samples has been instigated. We present the details of this approach as well as recent results for a range of materials system, including ${ }^{177} \mathrm{Lu}_{2-\mathrm{x}} \mathrm{Hf}_{\mathrm{x}} \mathrm{O}_{3}$. Here, Lu-177 is chosen as a surrogate for the radionuclides present in the actual waste because of its very short half-life (6.65 days), after which it decays to Hf.

In this presentation, we will examine both the evolution of structure and chemistry in connection with decaying radioactive isotopes with aberration corrected transmission electron microscopy. In particular we will focus on the results of transmission electron microscopy coupled with diffraction and related spectroscopies (EDS \& EELS) over a continuous time-series of data collected more than two half-lives. This talk highlights how we can apply transmission electron microscopy to study highly radioactive isotopes in small quantities. 
This work was supported by the laboratory directed research program at Los Alamos National Laboratory.

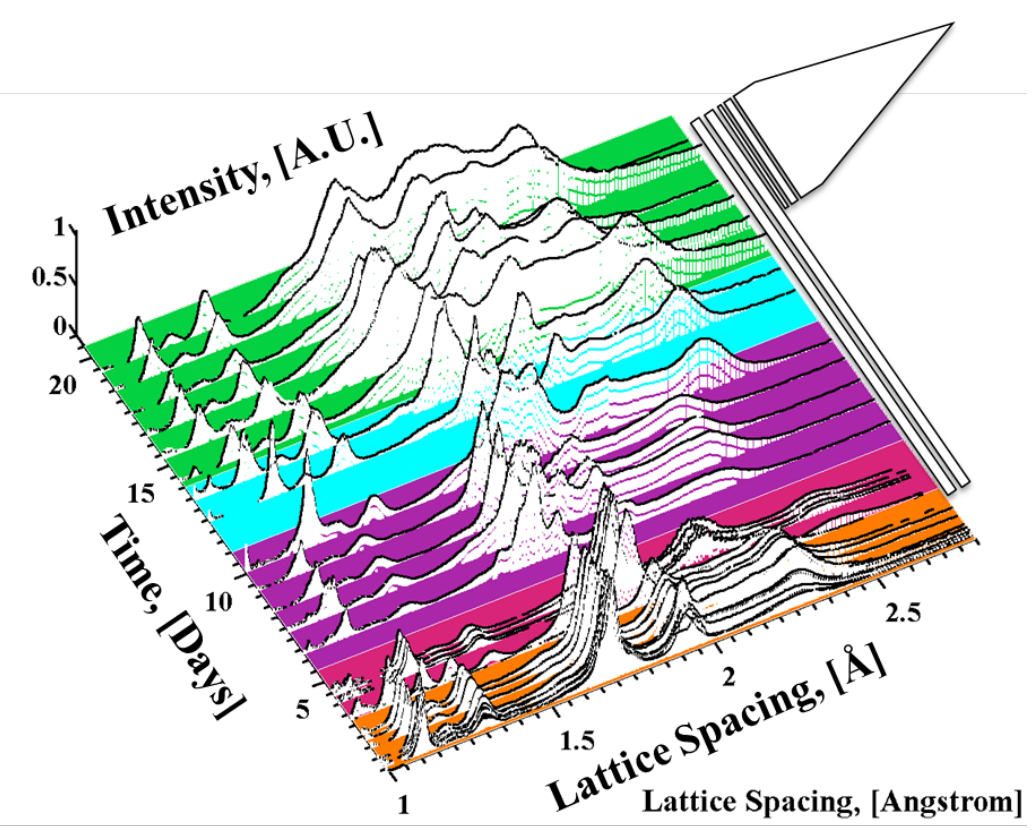

Figure 1: Above is an overview of the collected diffraction profiles tracking the decay of ${ }^{177} \mathrm{Lu}_{2-\mathrm{x}} \mathrm{Hf}_{\mathrm{x}} \mathrm{O}_{3}$. Collected diffraction profiles span the course of more than twenty days of transmuting $\mathrm{Lu}_{2} \mathrm{O}_{3}$ highlighting the structural evolution of transmuting oxide within an aberration corrected FEI Titan. These results highlight the potential use of in-situ based microscopy to study the structural decay and effects transmuting such as radioactive waste forms, such as those found at current nuclear repositories. 